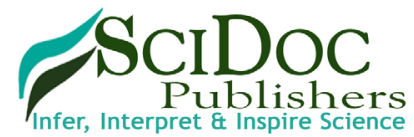

\author{
International Journal of Nano Studies \& Technology (IJNST) \\ ISSN:2167-8685
}

\title{
Current Advancements of the Smartphone - Based Point - Of - Carediagnosis
}

Review Article

Sun R, Chang YC, Wang LJ, Li L*

School of Mechanical and Materials Engineering, the Washington State University, Pullman, USA.

\section{Abstract}

The recent advancement in smartphone technology has triggered a wide range of smartphone point-of-care (POC) technology especially for the application of biomedical diagnosis and environmental detections. Modern smartphones with powerful processors and the functional cameras have been considered as the best candidate for miniaturization of the analytical instruments. Smartphones integrated with micro fluidic chips or optical components are capable of detecting specific biomarkers, antigens or pathogens in situ. The merits of smartphone-based POC devices, including cost efficiency, on-site detection, instantly obtaining diagnostic results, and transmittable data, make it grooming in medical and environmental diagnosis, especially in remote areas and at home. Therefore, smartphone-based POC technology could achieve decentralizing laboratory tests which fastens turnaround time and let doctors timely treat patients. In this review, we focus on paper-based and liquidbased smartphone POC devices of recent years to overviews current advancements.

Keywords: Smartphone; Point-Of-Care (POC) Diagnosis; Mobile Health; Paper Strip.

\section{Introduction}

Today, mobile phone technology with high-speed internet has been well-developed and been considered as the candidate technology for the next-generation handheld point-of-care (POC) devices with the function of transferrable diagnostic data. In 2015, it was estimated that there were 1.5 billion mobile phone users in developed countries, and 5.6 billion users in developing countries [1]. The high mobile penetration is extremely beneficial to the feasibility of smartphone-based POC diagnosis every where, especially in less developed countries, rural areas, and remote areas. These places lack diagnostic facilities and welltrained personnel. To improve healthcare systems in these under developed areas, rapid on-site diagnosis with data transmitting capability is in desperate need to immediately make medical decisions for patients. Recently, the concept of mobile health (mHealth) is active in many fields ranging from medical diagnostics to environmental detection. The camera of the smartphone stands out as the primary feature as a detection sensor. When the camera is integrated with the optical components, it can be used to sense and measure the changes in both color and optical intensity. Although human eyes can identify the difference in colors and brightness, we cannot quantify what we see into exact values. With the addition of optical components, such as diffraction gratings, micro lens, etc., the smartphone can be used as a visible light spectrum reader [2] or a microscopy [3]. Furthermore, the diagnostic results by smartphone-based devices can be uploaded through internet for doctors to make the treatment in time.

Smartphone-based POC diagnostics, with the advantages of rapid detection, transmittable data, and availability in rural areas, have had a great progress in recent years [4]. Conventional medical diagnosis typically requires sophisticated instruments, professionals or trained personnel to analyze samples. This regular diagnostic process works very well in developed counties or cities. However, it is very inconvenience to perform diagnostics in rural areas, especially in underdeveloped countries due to lacking the appropriate equipments and professionals. Currently, patients have to travel to the nearest laboratory and all testing samples from rural areas need to be transported to the nearest one in the city. There are several inconveniences, such as the preservation of the transported samples, particularly time-sensitive infected samples, patients enduring the painful situation for travelling, and back-and-forth time-consuming to know the results. These tedious procedures lead to the treatments delaying. For example,

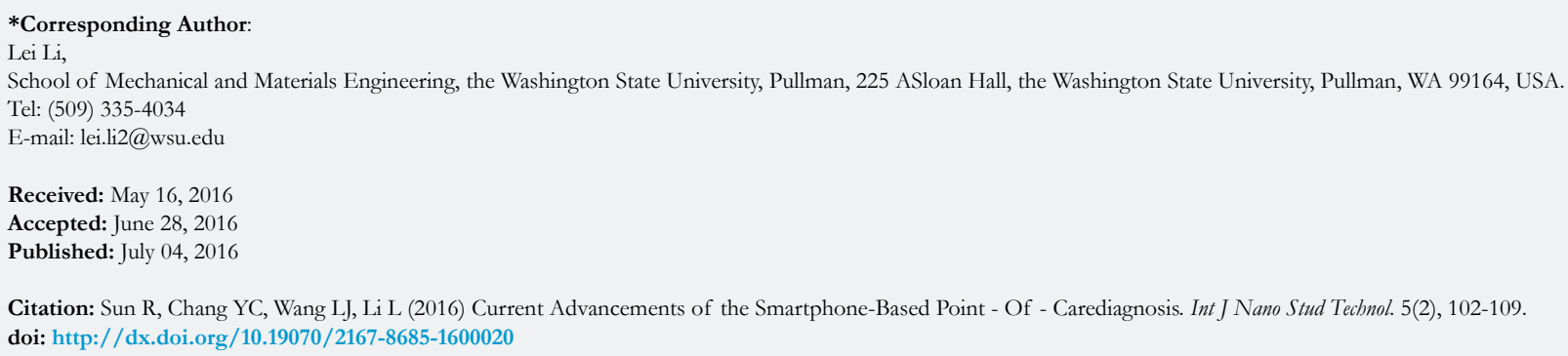

Copyright: $\mathrm{Li} \mathrm{L}^{\circ}$ 2016. This is an open-access article distributed under the terms of the Creative Commons Attribution License, which permits unrestricted use, distribution and repro duction in any medium, provided the original author and source are credited. 
the blood/urine test reports normally take several working days after collecting blood/urine samples from patients. In some emergent cases, the time-consuming diagnosis makes a lifeor-death difference [5]. To improve healthcare diagnostics and delivery, smartphone-based POC diagnosis attracts a number of researchers to achieve rapid diagnosis and timely readouts in situ.

A variety of POC diagnostic systems have been designed to be used in remote areas with untrained personnel. The development of smartphone-based POC diagnosis also makes it possible to monitor healthy conditions at home. For instance, patients with high cholesterol can measure their cholesterol concentration at home regularly and manage the data by smartphones. It will streamline their daily life, such as diet and exercise habits, etc. The regular monitoring can provide the doctors with more insight of these patients. The aim of this review paper underlines the current advancements of detection methods in smartphone-based POC systems. The camera of the smartphone is utilized in the detection process. The images were analyzed to diagnostic information as spectrum, color, and optical intensity and corresponded with the concentration of the analyses. In this paper, the smartphone based POC systems are introduced according to the testing types, including paper-based and liquid-based tests.

\section{Paper-Based Diagnostic Tests by Smartphone Based Devices}

Paper-based diagnostic tests are one of the most common clinical laboratory improvement amendment (CLIA)-waived tests. CLIAwaived tests refer to test systems that are cleared by the Food and Drug Administration (FDA) for at-home use, such as pregnancy urine strips, human immunodeficiency virus (HIV) strips, urine strips for urinalysis, thyroid hormone testing in whole blood, etc. In general, antibodies are embedded in paper strips which will trap target antigens or react with target reagents in the testing sample and result in color change. The main advantages of paper-based diagnostic tests are ultra low-cost (usually less than $\$ 0.50$ per test), fast (ranging from less than one minute to tens of minutes), and ease of use. Such paper-based diagnostic tests usually only show the positive/negative results by comparing to a control, but not quantitative results. Recently, with the assistance of a smartphone camera and applications (Apps), it has been demonstrated in laboratories that the shown color on the paper strips can be converted into actual values for quantification. In the biomedical diagnostics, samples such as blood, urine, saliva, and sweat are commonly tested because these substances are highly related to persons' health conditions and to infection detection. In the environmental detection, water quality is one of the important indexes for quality of life and agriculture. The paperbased method, for instance, PH strips, is a simple, low-cost, and rapid detection. However, the major challenges of the paper strip tests are low sensitivity and impossible to quantitatively analyze the data without laboratory equipments. Recently, some paperbased smartphone devices were developed for POC diagnosis to improve the quantification accuracy as well as the limit-ofdetection of paper strip diagnosis. They were used to detect the biomarkers or chemicals in fluids, such as blood, saliva, urine, sweat, water, etc. In this section, we will review the smartphone based POC application in paper-based diagnosis. The review is organized according to the sample types.

\section{Blood}

Blood tests are the most common medical diagnosis. In developing areas, paper strip tests for whole blood testing are the major diagnostic methods while lack of laboratory instruments. To develop quantitative analysis in remote areas, some research groups developed different smartphone-based devices. Once scu's group has conducted research of smartphone-based biosensors. They reported a paper-based smartphone system for monitoring cholesterol in blood, named as smart CARD (Figure. 1.1) [6]. Smart CARD can rapidly measure the cholesterol in the blood (within 60s) by analyzing the image of the test strip. They used the smartphone's flashlight to illuminate the testing area and used a diffuser (e.g. black polydimethylsiloxane (PDMS)) to reduce the consequences of a paper strip that may be misaligned. A smartphone camera was utilized to take images of the test strip. A smartphone App for the iPhone iOS platform was built to analyze Red, Green, and Blue (RGB) color on the Hue Saturation Lightness (HSL) Cylindrical-coordinate. The authors demonstrated the smart CARD by quantifying the cholesterol values in the blood tests, and compared values to the results from commercial hand held detector (Cardio Check PA) using the same samples. They got $3 \sim 5.5 \%$ difference between the results from these two readers. The accuracy increased to $1.8 \%$ if the cholesterol concentration is between $140 \mathrm{mg} / \mathrm{dl}$ to $400 \mathrm{mg} / \mathrm{dl}$. Later Once scu's group presented a smartphone-based accessory, which was named as vita AID (vitamin Au NP-based Immunoassay Device) (Figure 1.2) [7]. Vita AID was used to diagnose vitamin $\mathrm{D}$ level in the blood, and can achieve accuracy to be better than $15 \mathrm{nM}$.

In another group, Guan et al. have reported a portable smartphone diagnostic system based on a lab-made paper strip (Figure 1.3) [8]. It consists of a smartphone and a lab-made barcode-like paper strip. The built-in smartphone camera was used as a detector. An App was developed on the Android platform based on the identification of sample length on the test strip. " 0 " and " 1 " were represented "short" and "long" to indicate "positive" or "negative". A reference was preloaded in the app, which was obtained in statistical analysis. This smartphone-based barcodelike paper sensor was used to diagnose the blood typing (ABO/ $\mathrm{RhD}$ ) in the research. A number of 98 samples were detected. The results by this smartphone reader showed totally correct compared to the results from Red Cross Australia.

\section{Saliva}

Saliva is easy to obtain without any pain and it contains some biomarkers which can indicate some disease. Thus, it is another important body fluid in disease diagnostics. In Bae's research, a paper-based device was combined with a smartphone camera and light-emitting diode (LED) light source for alcohol concentration detection in saliva [9]. The modular detector comprises of a reflector for directing LED light, a diffuser for normalized lighting conditions, a plano convex lens to enhance the signal, and a sample holder (Figure 1.4). The paper strips are supplied by Alco-Screens (Chematics Inc., North webster, Indiana). The experiment was carried out by first dipping multiple strips into different concentrations of ethanol solution, along with a DI water sample as a negative control reference. The smartphone then took images of the paper strips in a controlled environment provided by the detection module. The images were then exported 
to a MATLAB program for RGB, and HSV analysis. The research group also developed an android-based App (Figure 1.5) to analyze a paper strip on-site. They showed the ability to detect the alcohol concentration ranging from $0 \%$ to $0.3 \%$.

Choi et al. reported a smartphone-based real-time measurement system for detecting human salivary cortisol [10]. This system included a smartphone, a holder, and a lateral flow immune strip. The authors used lab-made strips as test strips and used hue (color appearance parameters) and brightness in alab-developed App for the Android platform. They demonstrated the system's limit of detection (LOD) was $1 \mathrm{ng} / \mathrm{ml}$ with 0.7967 confidence of determination $\left(\mathrm{R}^{2}\right)$ using hue value. On the other hand, analyzing by using brightness values, $\mathrm{R}^{2}$ increased to 0.9837 . The authors claimed that this system predicted well if the sample's cortisol concentration was between 1 and $100 \mathrm{ng} / \mathrm{ml}$.

\section{Urine}

Urine is the frequently tested body fluid in medical diagnostics. Some smartphone devices were developed by different research groups to analyze urine. Choi et al. have developed a smartphonebased urine reagent reader to diagnose the leukocyte esterase (LE) and nitrite (NIT) in urine samples in the clinical emergency department (ED) [11]. This reader used the camera of the smartphone to analyze the data. ALED dimer was utilized to provide stable illumination, and a smartphone-based platform was used to aligne the smartphone with the sample (Figure 1.6). Commercial urine reagent strips were used for testing. Choi et al. demonstrated the high precision $(85.2 \%$ for LE and $97.5 \%$ for NIT) and the time efficiency ( 3 minutes) of this smartphone system compared to the commercial photometric analyzer ( 33 minutes without delivery time, 62 minutes with delivery time).

\section{Sweat}

Sweat is the easy-obtained body fluid in the daily life, especially while exercise. Once scu et al., developed a paper-based smartphone device to monitor the $\mathrm{pH}$ value and sodium plasma concentration in sweat (Figure 1.7) [12]. They designed a smartphone case and an insert chip as a diagnostic platform. The insert chip consists of an indicator strip (part of commercial plastic $\mathrm{pH}$ indicator strip), a reference strip (white plastic material) and a flash diffuser. The smartphone camera was used as a detector to take images of both test strips and the reference strip. In order to analyze the images, an app was created by analyzing hue in each image. The relationship between hue and $\mathrm{pH}$ was investigated, and the reference strip was used to correlate the real-time test results with lab-tested results. They defined the system's error less than 0.2 $\mathrm{pH}$ based on relevant $\mathrm{pH}$ value. In this research, sodium plasma concentration in the sweat and the $\mathrm{pH}$ value in both sweat and saliva were measured using this smartphone based accessory.

\section{Water}

Water quality monitoring is thought of as the measure of water conditions, including chemical, physical, and biological characteristics of water. Paper-based diagnostic tests are relatively easy and rapid, but cannot quantify the amount of analytes. Remcho's research group utilized a paper-based micro fluidic device to detect $\mathrm{Fe}^{2+}$ and $\mathrm{Cu}^{2+}$ content and concentration by a smartphone. Their paper-based device applied the capillary effect instead of pumps to reduce the complexity of the device, along with fabrication lead time [13]. They first pre-applied the assay reagents, potassium ferricyanide and potassium iodide, in the paper microfludic channels. Then, the solutions with different concentration of $\mathrm{Fe}^{2+}$ and $\mathrm{Cu}^{2+}$ ions were added into the paper channels. Different color and intensity were shown blue and red-brown color for $\mathrm{Fe}^{2+}$ and $\mathrm{Cu}^{2+}$, respectively (Figure 1.8). To quantify the analyte concentration related to color, a smartphone, along with RGB detection app (Color Assisted, FTLapps, Inc), was used. The authors demonstrated one of the simple paperbased micro fluidic colorimetric detection combined with a smartphone camera.

In Capitan-Vallvey's group, they introduced a paper-based micro fluidic device with a constant light source and a customized smartphone App to simultaneously detect $\mathrm{pH}$ value and Nitrite concentration. This design was specifically for drinking water which standard $\mathrm{pH}$ value should lie between 6.5 and 9.6 [14]. The recommended nitrite concentration is also noted by World Health Organization (WHO) with the value of no more than 3 milligrams per liter [15]. The paper-based micro fluidic device using filter papers was induced the channel paths by indelible ink. The indelible ink plays the role of hydrophobic wall for the capillary effect to direct the flow into the testing channels. On this paper-based micro fluidic platform, the dropped water sample started from a main chamber, and then flowed into 7 channels by capillary force. Each channel led to its own sub-chamber with $\mathrm{pH}$ and nitrite sensitive chemicals such as phenol red, chlorophenol red, sulphanilamide, N-1-naphthyl ethylenediamine dihydrochloride, and various solvents (Figure 1.9). However, the color changes cannot quantify the analytes by eyes. The same group also developed their own customized smartphone App for Hue, Saturation, and Value (HSV) analysis onan Android cell phone with a LED light as a consistent light source. The results displayed in the smartphone App and gave the $\mathrm{pH}$ value and nitrite concentration in milligrams per liter $(\mathrm{mg} / \mathrm{L})$.

\section{Liquid-based Diagnostic Tests by Smartphone Based Devices}

Although paper-base testing is very convenient for public individuals, the sensitivity is low and the strips usually just show positive/negative results. At clinical level to make clinical decision, liquid-based diagnostics are dispensable because of the ability of high sensitivity and low LOD. However, such high quality diagnostics need well-equipped laboratorial instruments and make turnaround time several working days to receive the reports. To improve the healthcare diagnostics and delivery, POC devices attract attentions in clinics and less-resource remote areas. Liquidbased POC smartphone devices are usually designed according to different optical sensing mechanisms by detecting absorbance at specific color, fluorescence intensity, or ultraviolet-visible (UVvis) spectrum. Based on different optical sensing methods, there are two major categories, fluorescence and colorimetric assays.

\section{Fluorescence Assays}

Fluorescence for labeling and detection is the most sensitive technology among the existing detection methods. Fluorescence has many practical applications in biochemistry and medicine, such as automated sequencing of DNA [16], fluorescence-activated 
Figure 1. Paper-based smartphone diagnostic systems: 1. the smart CARD accessory [6]; 2.thevitaAID accessory [7]; 3.the smartphone-based blood-typing detector [8]; 4.the smartphone detector [9]; 5.Paper strip detection application [9]; 6.the smartphone-based urine detector [11]; 7.the sweat detector [12]; 8.the paper-based microfluidic device [13]; 9.color detection area (left) and Android application user interface with quantify results post processing (right) [14].
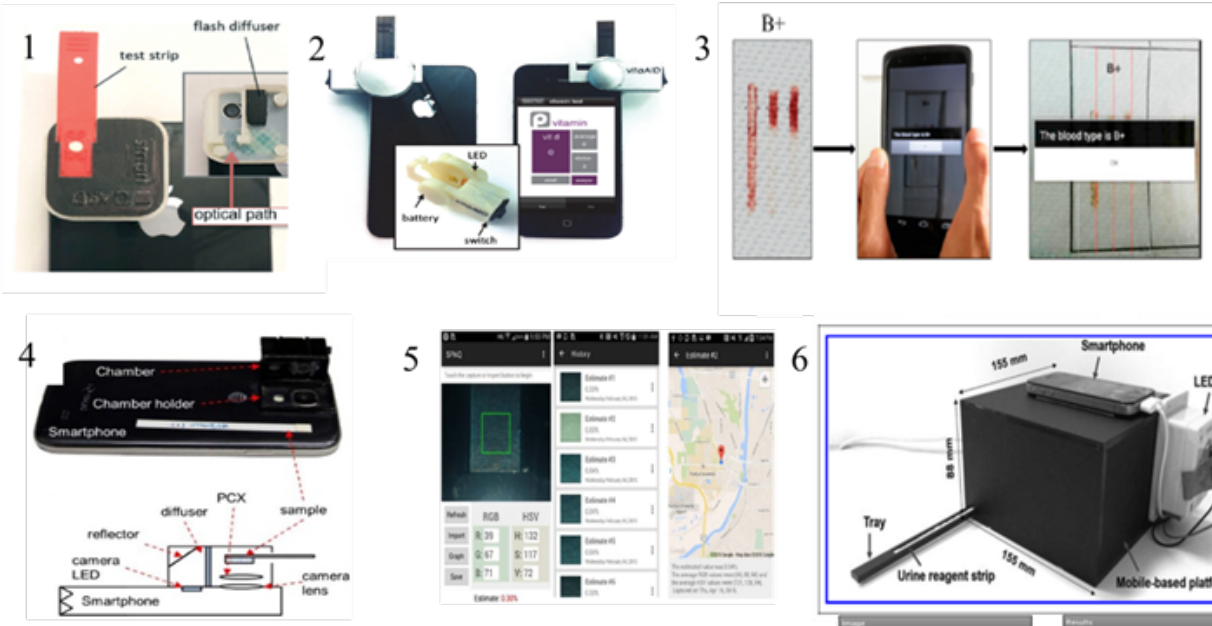

7

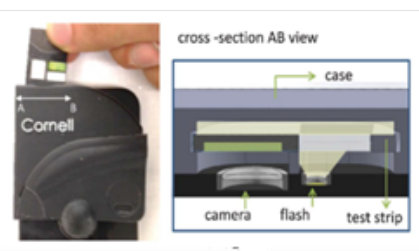

8

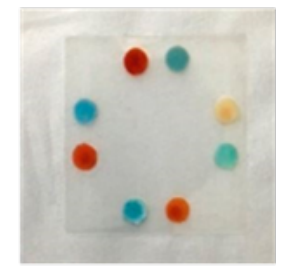

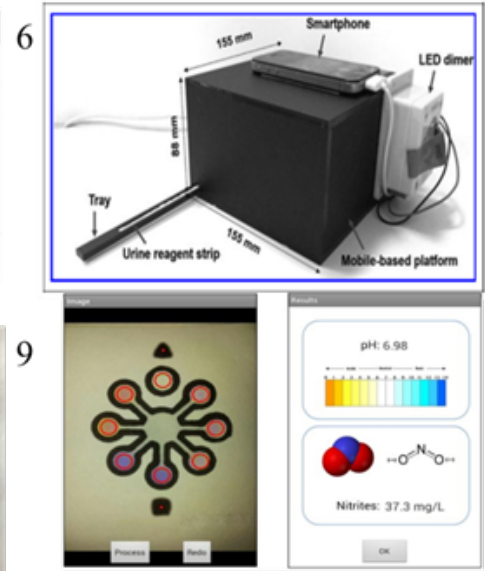

cell sorting [17], DNA detection [18], fluorescence image-guided surgery [19], and species altered fluorescence imaging [20]. Detecting fluorescence requires expensive, sensitive and specific instruments because fluorescence needs high intensity exciting radiation and fluorescence is normally week compared to exciting radiation. Moreover, tedious sample preparation, sophisticated operation skills, and time-consuming analysis are necessary in the laboratory. Most fluorescent probe faces quenching issue during such a long-time analysis. These limitations make the fluorescent medical diagnosis costly. Since the concept of POC has been drawn much attention, some researchers are devoted to develop smartphone-based fluorescent platform for fluorescent detection.

Breslauer et al., has studied a smartphone-based POC system for many years. In 2009, they developed a mobile phone based clinical fluorescent microscopy to take image of P. falciparum-infected, sickle red blood cells, and M. tuberculosis-infected sputum samples in a bright field and a fluorescence field, respectively (Figure 2.1) [21]. The system's spatial resolution is around 1.2 $\mu \mathrm{m}$. Though they conducted the image analysis using a laptop computer instead of developing a smartphone app, their research still played a major role in the development of smartphone-based system.

Coskun et al. conducted a research in analyzing fluorescent assays for albumin detection in urine samples on a smartphone system, named Albumin Tester [22]. This system was shown in Figure 2.2. A laser diode was used as an excitation source; a disposable tube was used as sample container, and a smartphone camera was used for imaging capture. The authors claimed that Albumin Tester achieved the detection limit less than $10 \mu \mathrm{g} / \mathrm{mL}$, and it took around $5 \mathrm{mins}$ per test. The authors mentioned that this system can be modified to measure other biomarkers, such as creatinine.
Zhu et al. developed an imaging cytometry platform attached to a smartphone to measure the density of white blood cells (WBCs) [23]. Figure 2.3 shows the pictures of this system. In this design, 8 LEDs were used as light source; a plastic filter was used to decrease the amount of scattered light, a plano-convex lens was used to decrease the focal length of the system. Fluorescently labeled WBCs samples were inserted into the top chamber and images were taken by this system. A custom-developed App was developed to automatically count the number of WBCs and calculated the density of WBCs. Different WBC concentrations (from 3000 per $\mu \mathrm{L}$ to 12000 per $\mu \mathrm{L}$ ) were tested and compared with a commercial hematology analyser (Systemx KN21). This smartphone system obtained an absolute error within $7 \%$ in the standard test and attained a correlation coefficient around 0.98 between this Smartphone based system and commercial analyzer.

\section{Colorimetric Assays}

In colorimetric assays, enzyme-linked immune sorbent assay (ELISA) is the most common laboratorial performed assay. ELISA requires the specific antibodies to detect antigens and then adds color reagents to produce the detectable color signal [24]. However, to read the values of the optical density for quantification, the spectrophotometer with well-trained professionals is necessary. Surface Plasmon resonance (SPR) has appeared to be an attractive label-free detection method for measuring adsorption of analytes on planar metal surface, typically gold or silver, or on the surface of metal nanoparticles. The refractive index of the metal surface changed before and after conjugating analytes [25]. Therefore, SPR is proposed as the newly sensing method for label free immunoassays [26]. 
ELISA: Li's group developed a smartphone optosensing platform (SOP) for neurotoxins detection by ELISA assays [27]. Their SOP device utilized adigital versatile disc (DVD) as a low-cost diffraction grating to make an affordable and portable spectrometer. Compared to commercial diffraction grating which is $\$ 75 \mathrm{USD}$, the DVD grating only costs 2.5 cents for each piece. The DVD grating is integrated with a $3 \mathrm{D}$ printed smartphone holder and a cuvette holder that is connected with a light source in Figure 2.4. The tested sample solution was added in the transparent cuvette, and then place e in the cuvette holder. Images were taken by the smartphone camera and further analyzed the absorbance at the specific wavelength. The LOD achieved $2.9 \mathrm{nM}$ for the paraxon neurotoxins which is very close to the LOD of the commercial spectrometer. The researcher estimated further massive production of the whole SOP platform (excluding smartphone) and cost below $\$ 50$ USD. With comparative performance with the commercial laboratory spectrometer but affordable cost, SOP can be brought to marketplace and remote areas.

Berg et al. developed a smartphone-based platform for POC testing of ELISA (Figure 2.5) [28]. They utilized 24 LEDs as illumination sources and homogenized the light using two layers of plastic plates. They used 96 fibers under each well of a 96-well micro plate to increase the field of view (FOV) and to decrease the image aberration. The transmitted illumination densities were measured using smartphone camera. The authors tested 4 types of Ig G by ELISA assays, including mumps, measles, HSV-1, and HSV-2. They compared these results with the FDA-approved commercial ELISA reader. They obtained a high accuracy of $99.6 \%, 98.6 \%, 99.4 \%$ and $99.4 \%$ for these samples, respectively.

SPR: Filho et al., utilized a smartphone front-facing camera and active-matrix organic light emitting diodes (AMOLED) to detect SPR for measuring analytes [29]. The optical sensing chip was composed of four layers of materials. The first layer was Cyclic Olefin Copolymer (COC) manufacture by TOPAS Advanced Polymers Inc. The second layer was gold film, and the remaining layers depend on the type of samples. In the bottom, there were two diffractive optical coupling elements (DOCE), one facing the light source and the other aligned with the front facing camera.

Figure 2. Liquid-based smartphone diagnostic systems: 1. The mobile phone fluorescent microscopy [21]; 2. Albumin Tester [22]; 3. the pictures of smartphone based cytometry platform [23]; 4. SOP device [28]; 5 . ELISA based 96 well smartphone system [29].

1
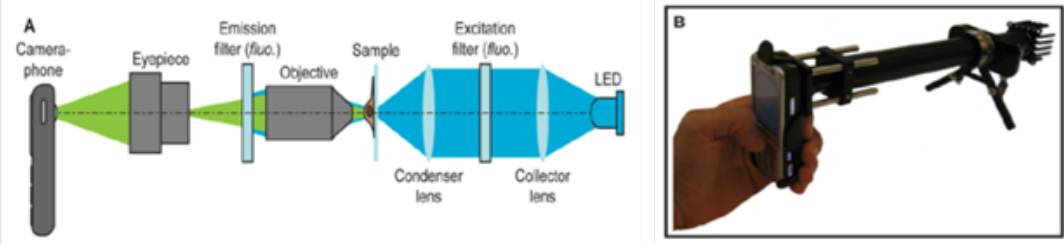

2

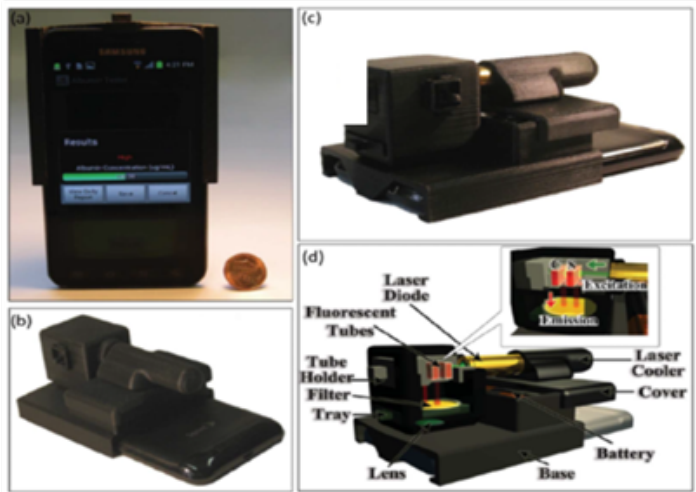

3

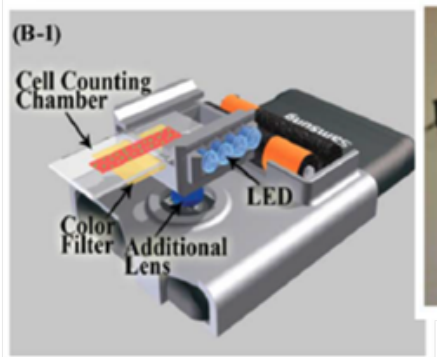

4

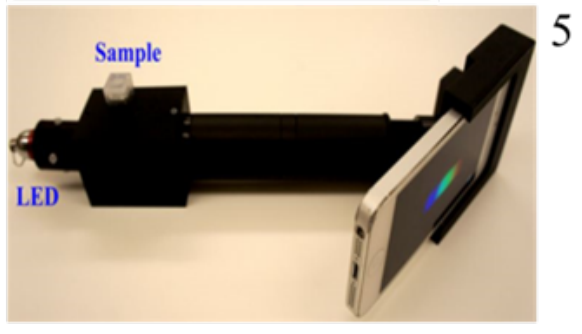

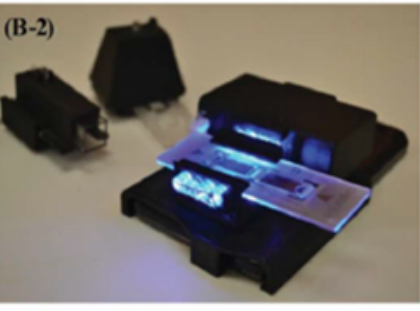

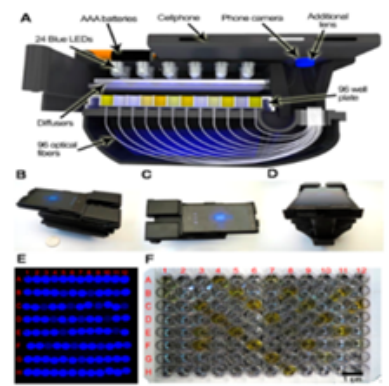


The tested samples included $\mathrm{H}_{2} \mathrm{O}, \mathrm{NaClO}$, bovine serum albumin (BSA) solution, and $\mathrm{NaCl}$ aqueous solution. A flow of solution was injected into a sensory located in the device. Simultaneously, the smartphone AMOLED screen displayed different colored images acting as a light source while the camera readout was converted into its respective SPR curves. The sensitivity of this smartphone SPR system reached as low as $10^{-5}$ RIU (refractive index unit). They concluded that the results were promising compared to lab-grade SPR type instruments.

In Filippini's research group, SPR was utilized for $\beta_{2}$ micro globulin $\left(\beta_{2} \mathrm{M}\right)$ Detection [30]. A device composed of poly dimethyl siloxane (PDMS), gold coating surface, and two optical couplers were fabricated. The PDMS optical couplers were properly designed in order to acquire the proper angle-resolved SPR signal. The sensitivity was first determined through a series of intensity vs. illuminating angle plot tests with both red and green illumination. Then it was calculated to be $11 \times 10^{-3}$ RIU per degree. The device was combined with a commercial test chip Biacore CM5 (Biacore) to detect $\beta$, microglobulin. The team was able to achieve detection of a relatively low concentration with the LOD $0.1 \mu \mathrm{g} / \mathrm{mL}$. The group concluded that it will perform in this range within the clinical application requirement. Furthermore, they proved the concept of the disposability and reliability of an SPR based system.

\section{Bioluminescence and Chemiluminescence}

Bioluminescence and chemiluminescence are self-emitting light through chemical reaction. Bioluminescence could detect bioluminescent biomarkers, such as bioluminescent bacteria.
Similarly, chemiluminescence is based on the emitted photons produced during the chemical reaction. For example, luminol is a common chemical agent and activated by oxidant and catalyst to emit visible light [31]. The following will introduce smartphonebased devices for colorimetric assays.

Cevenini et al., demonstrated the smartphone-based bioillumination toxicity detector [32]. They designed cell phone accessories, including a smartphone adaptor, droppers, reservoirs, and a sliding lid, to make this system become a standalone detector. A toxicity App (Tox-App) was developed using Python Imaging Library on an Android platform. The cell viability and the toxicity level (safe if $100-80 \%$, harmful if $79-30 \%$ and highly if $<30 \%$ ) of the sample were obtained. This system took less than 30 minutes for each test. The embryonic kidney Hek293T cells (used as "sentinel cells") were tested in this research. The results were compared with test results from a Var-ioskan Flash multimode reader. The coefficient of variation was $15 \%$ using this standalone detector.

Roda et al., investigated a smartphone-based luminometer to detect enzyme-coupled chemiluminescence samples, such as lactate in artificial sweat and saliva [33]. The LOD of this system is about $0.1 \mathrm{mmol} / \mathrm{L}$ for sweat detection and $0.5 \mathrm{mmol} / \mathrm{L}$ for saliva detection. We summarized more liquid-based smartphone diagnostic devices in Table 1.

\section{Conclusion}

This review summarized and discussed current advancement of smartphone-based POC diagnostic devices. The majority of

Table 1. Liquid-based smartphone POC diagnostic devices.

\begin{tabular}{|c|c|c|c|c|}
\hline Method & Sample & $\begin{array}{l}\text { Limitation of Detection (LOD) } \\
\text { and accuracy }\end{array}$ & Author & Year \\
\hline fluorescent & $\begin{array}{l}\text { 1.Fluorescent bead } \\
\text { 2.White blood cell } \\
\text { 3. White blood cell }\end{array}$ & $\begin{array}{l}\text { 1. Spatial resolution: around } 10 \mu \mathrm{m} \text {. } \\
\text { 2.field-of-view : around } 81 \mathrm{~mm} 2\end{array}$ & Zhu et al.[i] & 2010 \\
\hline fluorescent & $\begin{array}{c}\text { hepatitis } \\
\text { B virus (HBV) plasmid sam- } \\
\text { ples }\end{array}$ & Accuracy range from $85 \%$ to $100 \%$. & Lee et al.[ii] & 2011 \\
\hline fluorescent & white blood cells & spatial resolution: $\sim 2 \mu \mathrm{m}$ & Zhu et al.[iii] & 2011 \\
\hline fluorescent & Escherichia coli (E. coli) & $\begin{array}{c}\text { LOD: } \\
\text { around } 5 \text { to } 10 \mathrm{cfu} / \mathrm{mL}\end{array}$ & Zhu et al.[iv] & 2012 \\
\hline ELISA/Spectrum & $\begin{array}{l}\text { 1.Interleukin-6 (IL-6) in serum } \\
\text { samples } \\
\text { 2.Ara h } 1 \text { (in Cookie Samples) }\end{array}$ & $\begin{array}{c}\text { LOD: } \\
\text { 1.IL-6: } 2 \mathrm{pg} / \mathrm{mL} \\
\text { 2.Ara h1: } 0.09 \sim 0.56 \mathrm{ppm}\end{array}$ & Long et al.[v] & 2014 \\
\hline ELISA/Spectrum & $\begin{array}{l}\text { 1. Paraoxon in phosphate } \\
\text { buffer saline (PBS) } \\
\text { 2. Paraoxon in tap water }\end{array}$ & $\begin{array}{l}\text { 1. } 4.3 \mathrm{nM} \\
\text { 2. } 3.2 \mathrm{nM}\end{array}$ & Wang et al.27 & 2016 \\
\hline $\begin{array}{c}\text { ELISA } \\
\text { /Colorimetric }\end{array}$ & $\begin{array}{l}\text { 1. Mumps HSV } \\
\text { 2. Measles } \\
\text { 3. HSV } 1 \\
\text { 4. HSV } 2\end{array}$ & $\begin{array}{l}\text { Accuracy: } \\
\text { 1. } 99.6 \% \\
\text { 2. } 98.6 \% \\
\text { 3. } 99.4 \% \\
4.99 .4 \% \\
\end{array}$ & Berg et al.28 & 2015 \\
\hline $\begin{array}{l}\text { Bioluminescence; } \\
\text { chemiluminescence }\end{array}$ & $\begin{array}{l}\text { 1. bile acids; } \\
\text { 2. cholesterol }\end{array}$ & $\begin{array}{l}\text { LOD: } \\
\text { 1. } 0.5 \mu \mathrm{mol} / \mathrm{L} \text { for bile acids } \\
\text { 2. } 20 \mathrm{mg} / \mathrm{dL} \text { for cholesterol }\end{array}$ & Rodaet al.[vi] & 2014 \\
\hline
\end{tabular}


diagnostic technologies were covered in this review, categorized by paper-based and liquid-based tests. In general, liquid-based tests achieve higher sensitivity and accuracy compared to the paper-based methods. However, the paper-based tests are CLIAwaived for public individual and not limited in the laboratory.

In section 2, we introduced paper-based smartphone diagnostic devices. With the rapid development of smartphones, paperbased smartphone sensors can diagnose samples quantitatively. Since CLIA-waived paper strip tests are very convenient and rapid in field and at home, the ability to quantify analyte amounts by a simple smartphone system (smartphone and its accessory) is helpful for individuals. The paper-based diagnostic devices combined with a smartphone provide a low cost, accurate, and user-friendly interface for public consumers.

In section 3, liquid-based smartphone diagnostic devices were introduced. The fluorescent assays are the most sensitive method for low-concentration biomarkers. ELISA is the most common diagnostic method. SPR is the label-free method to diagnose biomarkers. Although bioluminescence and chemiluminescence methods are not highly sensitive, especially for low concentration, these methods don't require addition of light source due to selfillumination.

In summary, the smartphone-based POC systems have the merits of decentralizing laboratory testing and transmittable diagnostic results via internet. In the future, the smartphone-based POC systems could support clinician to perform the diagnostic tests in situ, let the physician take a picture of an X-ray on-site, let patients get the diagnostic results immediately, and allow doctors to treat patients timely. With the development of mobile technologies, the gap between inpatient and outpatient care could also be close by the smartphone-based POC systems.

\section{References}

[1]. Gallegos D, Long KD, Yu H, Clark PP, Lin Y, George S, et al., (2013) Labelfree biodetection using a smartphone. Lab Chip 13(11), 2124-2132.

[2]. Smith ZJ, Chu K, Espenson AR, Rahimzadeh M, Gryshuk A, Molinaro M, et al., (2011) Cell-phone-based platform for biomedical device development and education applications. PloS one 6 (3): e17150.

[3]. Gopinath SC, Tang TH, Chen Y, Citartan M, Lakshmipriya T (2014) Bacterial detection: From microscope to smartphone. Biosensors and Bioelectronics 60: 332-342.

[4]. Xu, X, Altug Akay, Huilin Wei, ShuQi Wang, Belinda Pingguan-Murphy, Björn-Erik Erlandsson, et al., (2015) Advances in smartphone-based pointof-care diagnostics. Proceedings of the IEEE 103(2): 236-247.

[5]. Gubala V, Harris LF, Ricco AJ, Tan MX, Williams DE (2011) Point of care diagnostics: status and future. Analytical chemistry 84(2): 487-515.

[6]. Oncescu V, Mancuso M, Erickson D (2014) Cholesterol testing on a smartphone. Lab on a Chip 14(4): 759-763.

[7]. Lee S, Oncescu V, Mancuso M, Mehta S, Erickson D (2014) A Smartphone platform for the quantification of vitamin D levels. Lab on a Chip 14 (8): 1437-1442.

[8]. Guan L, Tian J, Cao R, Li M, Cai Z, Shen W (2014) Barcode-like paper sensor for smartphone diagnostics: An application of blood typing. Analytical chemistry. 86(22): 11362-11367.

[9]. Jung Y, Kim J, Awofeso O, Kim H, Regnier F, et al., (2015) Smartphonebased colorimetric analysis for detection of saliva alcohol concentration. Applied optics 54(31): 9183-9189.

[10]. Seoyeon Choi, Soocheol Kim, Jung-Sik Yang, Jung-Hyun Lee, Chulmin Joo, et al., (2014) Real-time measurement of human salivary cortisol for the assessment of psychological stress using a smartphone. Sensing and BioSensing Research 2: 8-11.

[11]. Choi K, Chang I, Lee JC, Kim do K, Noh S, et al., (2016) SmartphoneBased Urine Reagent Strip Test in the Emergency Department.Telemedicine and e-Health 22(6): 1-7.
[12]. Oncescu V, O'Dell D, Erickson D (2013) Smartphone based health accessory for colorimetric detection of biomarkers in sweat and saliva. Lab on a Chip 13(16): 3232-3238.

[13]. Myra T.Myra T. Koesdjojo, Sumate Pengpumkiat, Yuanyuan Wu, Anukul Boonloed, Daniel Huynh (2015) Cost Effective Paper-Based Colorimetric Microfluidic Devices and Mobile Phone Camera Readers for the Classroom. Journal of Chemical Education 92(4): 737-741.

[14]. Lopez-Ruiz N, Curto VF, Erenas MM, Benito-Lopez F, Diamond D, et al., (2014) Smartphone-based simultaneous $\mathrm{pH}$ and nitrite colorimetric determination for paper microfluidic devices.Analytical chemistry 86(19): 95549562.

[15]. World Health Organization (2003) Nitrate and nitrite in drinking-water: Background document for development of WHO Guidelines for Drinkingwater Quality. WHO Press, Geneva, Switzerland. 1-31.http://www.who.int/ water_sanitation_health/dwq/chemicals/nitratenitrite2ndadd.pdf.

[16]. Prober JM, Trainor GL, Dam RJ, Hobbs FW, Robertson CW, et al., (1987) A system for rapid DNA sequencing with fluorescent chain-terminating dideoxynucleotides. Science 238(4825): 336-341.

[17]. Fu AY, Spence C, Scherer A, Arnold FH, Quake SR (1999) A microfabricated fluorescence-activated cell sorter. Nature biotechnology 17(11): 11091111.

[18]. Zhao X, Tapec-Dytioco R, Tan W (2003) Ultrasensitive DNA detection using highly fluorescent bioconjugated nanoparticles. Journal of the American Chemical Society 125(38): 11474-11475.

[19]. Schaafsma BE, Mieog JS, Hutteman M, van der Vorst JR, Kuppen PJ, et al., (2011) The clinical use of indocyanine green as a near-infrared fluorescent contrast agent for image-guided oncologic surgery. Journal of surgical oncology 104(3): 323-332.

[20]. Klucken J, Outeiro TF, Nguyen P, McLean PJ, Hyman BT (2006) Detection of novel intracellular $\alpha$-synuclein oligomeric species by fluorescence lifetime imaging. The FASEB Journal 20(12): 2050-2057.

[21]. Breslauer DN, Maamari RN, Switz NA, Lam WA, Fletcher DA (2009) Mobile phone based clinical microscopy for global health applications. PloS one 4(7): e6320.

[22]. Coskun AF, Nagi R, Sadeghi K, Phillips S, Ozcan A (2013) Albumin testing in urine using a smart-phone. Lab on a Chip 13(21): 4231-4238.

[23]. Zhu H, Sencan I, Wong J, Dimitrov S, Tseng D, et al., (2013) Cost-effective and rapid blood analysis on a cell-phone. Lab on a Chip 13(7): 1282-1288.

[24]. Clark MF, Adams AN (1977) Characteristics of the microplate method of enzyme-linked immunosorbent assay for the detection of plant viruses. Journal of general virology 34(3): 475-483.

[25]. Pattnaik P (2005) Surface plasmon resonance. Applied biochemistry and biotechnology 126(2): 79-92

[26]. Rich RL, Myszka DG (2007) Higher-throughput, label-free, real-time molecular interaction analysis. Analytical biochemistry 361(1): 1-6.

[27]. Wang LJ, Chang YC, Ge X, Osmanson A, Du D, et al., (2016) Smartphone Optosensing Platform Using a DVD Grating to Detect Neurotoxins.ACS Sensors 1(4): 366-373

[28]. Berg B, Cortazar B, Tseng D, Ozkan H, Feng S, et al., (2015) Cellphonebased hand-held microplate reader for point-of-care testing of enzymelinked immunosorbent assays. ACS nano 9(8): 7857-7866.

[29]. de Souza Filho CA, Lima AMN, Neff H (2014) Smartphone based, portable optical biosensor utilizing surface plasmon resonance. InInstrumentation and Measurement Technology Conference (I2MTC) Proceedings, 2014 IEEE International. 890-895.

[30]. Preechaburana P, Gonzalez MC, Suska A, Filippini D (2012) Surface plasmon resonance chemical sensing on cell phones. Angewandte Chemie International Edition 51(46): 11585-11588.

[31]. Hastings J, Wilson T (1976) Bioluminescence and chemiluminescence. Photochemistry and photobiology 23(6): 461-473.

[32]. Cevenini L, Calabretta MM, Tarantino G, Michelini E, Roda A (2016) Smartphone-interfaced 3D printed toxicity biosensor integrating bioluminescent "sentinel cells". Sensors and Actuators B: Chemical 225: 249-257.

[33]. Roda A, Guardigli M, Calabria D, Calabretta MM, Cevenini L, et al., (2014) A 3D-printed device for a smartphone-based chemiluminescence biosensor for lactate in oral fluid and sweat. Analyst 139(24): 6494-6501.

[34]. Zhu H, Yaglidere O, Su TW, Tseng D, Ozcan A (2011) Cost-effective and compact wide-field fluorescent imaging on a cell-phone. Lab on a Chip 11(2): 315-322.

[35]. Lee D, Chou WP, Yeh SH, Chen PJ, Chen PH (2011) DNA detection using commercial mobile phones. Biosensors and Bioelectronics 26(11): 43494354.

[36]. Hongying Zhu, Sam Mavandadi, Ahmet F. Coskun, Oguzhan Yaglidere, Aydogan Ozcan (2011) Optofluidic fluorescent imaging cytometry on a cell phone. Analytical chemistry 83(17): 6641-6647.

[37]. Zhu H, Sikora U, Ozcan A (2012) Quantum dot enabled detection of Escherichia coli using a cell-phone. Analyst 137(11): 2541-2544. 
[38]. Long KD, Yu H, Cunningham BT (2014) Smartphone instrument for portable enzyme-linked immunosorbent assays. Biomedical optics express 5(11): 3792-3806.

[39]. Roda A, Michelini E, Cevenini L, Calabria D, Calabretta MM, et al., (2014) Integrating biochemiluminescence detection on smartphones: mobile chemistry platform for point-of-need analysis. Analytical chemistry 86(15): 7299 7304 . 